\title{
Synchrotron radiographic studies of ultrasonic melt processing of Metal Matrix Nano Composites
}

\author{
Wajira Mirihanage $^{1,}{ }^{*}$, Wenwu Xu ${ }^{1}$, Javier Tamayo-Ariztondo $^{2}$, Dmitry Eskin $^{2}$, Mirian Garcia-Fernandez $^{3}$ \\ Prakash Srirangam ${ }^{4}$, Peter Lee ${ }^{1}$ \\ ${ }^{1}$ School of Materials, The University of Manchester, Manchester M13 9PL, UK \\ ${ }^{2}$ Brunel Centre for Advanced Solidification Technology, Brunel University London, Uxbridge UB8 3PH, UK \\ ${ }^{3}$ Diamond Light Source, Didcot OX11 ODE, UK \\ ${ }^{4}$ WMG, University of Warwick, Coventry CV4 7AL, UK \\ * Corresponding author: email: wajira.mirihanage@manchester.ac.uk phone: +44 (0)1235 567848
}

\begin{abstract}
Fast synchrotron radiography was used to investigate ultrasonic cavitation bubble formation and their dynamics during liquid metal processing of $\mathrm{Al}-\mathrm{Cu}$ metal matrix nano composites (MMNC) in comparison with conventional alloys. The experimental observations showed enhanced cavitation potential in MMNC melts, due to the presence of $\mathrm{Al}_{2} \mathrm{O}_{3}$ nano particles which believed to be acting as heterogeneous nuclei for bubble formation. Quantitative image analysis demonstrates that the addition of nano particles increases melt agitation partially, while introducing higher flow velocity variations across the melt. This suggests that the presence of nano particles may substantially alter propensity for ultrasonic treatment effects during solidification processing of MMNCs.
\end{abstract}

\section{Highlights}

- Fast X-ray imaging of ultrasound cavitation in Al-Cu metal matrix nano composite melts.

- Nano particles significantly enhance the ultrasonic cavitation bubble formation potential.

- Higher flow velocity variation across the melt with increased cavitation activity was found from the experimental observations.

Key words: metal matrix nano composites, synchrotron radiography, ultrasonic cavitation, aluminium

\section{Introduction}

Metal matrix nano composites (MMNC) have emerged recently as a next generation light material for transport applications, with a promise to reduce carbon emissions with enhanced specific strengths [1]. MMNCs are metallic alloys with embedded extrinsic nano particles (NP) which act like nanoprecipitates and offer a potential step change in mechanical properties via Orowan strengthening [2]. 
Although MMNC production through mechanical forming has demonstrated significant mechanical property improvements [1], production costs are deemed to be too high. Solidification routes are more cost effective, but due to NP agglomeration and further segregation/pushing by solid-liquid interphase, ordinary casting methods found to be ineffective [3]. Ultrasonic melt processing offers a promising approach to de-agglomerate NP clusters, facilitating better NP incorporation into the primary phase via reduced pushing $[4,5]$. Ultrasonic processing has also been employed in the metal casting industry as a popular technique to degas molten metal to control porosity and enhance grain refinement during solidification $[4,6]$. The cavitation bubble sizes and their number density are found to play an important role in governing all of these aspects [4]. Hence, insights into the dynamics associated with ultrasonic treatment are crucial to optimize solidification processing of molten MMNCs. Although NPs are recognised to alter strength in the solid, and viscosity in the liquid [7, 8], their influence on ultrasound treatment has not been studied yet and is required to facilitate the optimization of MMNC processing via solidification routes.

High speed optical imaging based analysis of ultrasound cavitation in pure water has been well documented (e.g. ref. [9]), but is not viable for opaque molten alloys. Though, in situ X-radiography has been used to study a range of phenomena associated with solidification processes of metallic alloys [10, $11,12,13,14,15]$, very few in situ X-ray experimental studies were reported to date on cavitation interactions in molten and solidifying metals $[16,17]$. Most of these studies had limited time resolution, except in very recent research [18], where ultra-fast shock wave effects related to ultrasound sonication has been reported. Recently, small angle X-ray scattering (SAXS) was also employed to investigate the cavitation bubble nucleation phenomena in Al-Cu melts [19]; the limitation with this study was that once cavitation bubbles were nucleated, they were quickly grown to sizes which is beyond the capability of SAXS to resolve. Further, to the best of authors' knowledge, no direct in situ experimental observation has been reported to evaluate the ultrasonic cavitation driven bubble formation in molten metal with nano particles. Here, we report in situ synchrotron X-ray observations of ultrasound cavitation in molten aluminium with NPs (MMNCs).

\section{Experimental details}

The composition of the MMNC samples was Al-10wt\%Cu with 0.05 wt $\% \mathrm{Al}_{2} \mathrm{O}_{3}$ NP (average size $80 \mathrm{~nm}$ ). Initially, NPs were wrapped in thin aluminium foil and added to a $1 \mathrm{~kg}$ batch of the molten Al- 
$10 w t \% \mathrm{Cu}$ alloy during mechanical stirring with a Ti impeller, followed by ultrasonic processing for $15 \mathrm{~min}$ (17.5 kHz, Nb horn). The mixture was then poured at $700{ }^{\circ} \mathrm{C}$ into a $10 \mathrm{~mm}$ diameter, $120 \mathrm{~mm}$ long cylindrical steel mould. A separate set of reference samples were also prepared trough to the same processing but without adding NPs. Samples were then machined to have a tight fit into the bottom part of the boron nitride (BN) crucible, $1 \mathrm{~mm}$ thin, $3 \mathrm{~mm}$ wide and $4 \mathrm{~mm}$ high cavity (see Figure 1.a). The crucible was placed in a resistance heating furnace [12] with the thinner bottom part of the crucible positioned between two X-ray windows $(10 \mathrm{~mm} \times 10 \mathrm{~mm})$. The sample thickness direction vector was parallel to the synchrotron X-ray beam (Figure 1b). A $30 \mathrm{kHz}$-Hielscher® UP50H ultrasonic processor with $0.5 \mathrm{~mm} \mathrm{Ti}$ sonotrode tip was fixed to a remote controlled vertically movable rail, in order to move the sonotrode tip into a repeatable position inside the molten metal within $<10 \mu \mathrm{m}$ accuracy. A low rate Ar supply was also directed to the sample top surface to minimise the oxidation during the experiments.

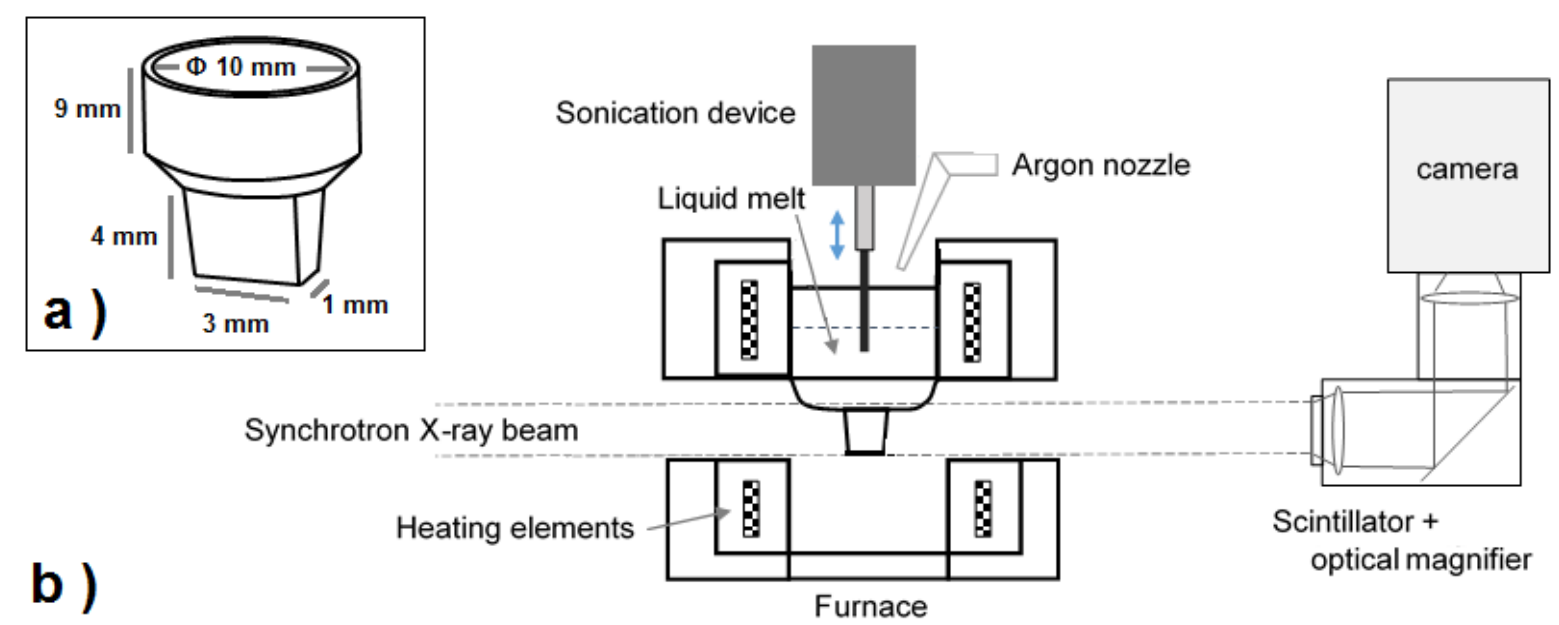

Figure 1. a) Schematic shape of crucible used (no to scale, external dimensions are shown). b) Experimental configuration.

During the experiments in Diamond-Manchester branchline (I13-2) at Diamond Light Source, UK; the crucible was heated to $700 \pm 5^{\circ} \mathrm{C}$, at the rate of $\sim 0.5^{\circ} \mathrm{C} / \mathrm{s}$, with subsequent $\sim 600 \mathrm{~s}$ temperature homogenisation holding period. The oscillating sonotrode tip was immersed approx. $4 \mathrm{~mm}$ into the molten metal for a $1.0 \mathrm{~s}$ sonication cycle, where power is applied for $0.6 \mathrm{~s}$ and then turned off for $0.4 \mathrm{~s}$. A pink synchrotron X-ray beam was used for imaging (with the $1^{\text {st }}$ mode at $\sim 16 \mathrm{keV}$ [20]). On the detector side, a CdWO4 scintillator was coupled to a Pco.Edge ${ }^{\circledR}$ camera through an optical magnification system. The 
imaging field of view was $\sim 4.2 \times \sim 3.5 \mathrm{~mm}^{2}$ with $1.6 \mu \mathrm{m}$ spatial resolution. Radiographs were collected at $63 \mathrm{~Hz}$ with $10 \mathrm{~ms}$ exposure time, which provided $\sim 0.016 \mathrm{~s}$ nominal temporal resolution. For all the experiments presented here, top edge of the images were $\sim 3 \mathrm{~mm}$ below the sonotrode tip.

\section{Results and discussion}
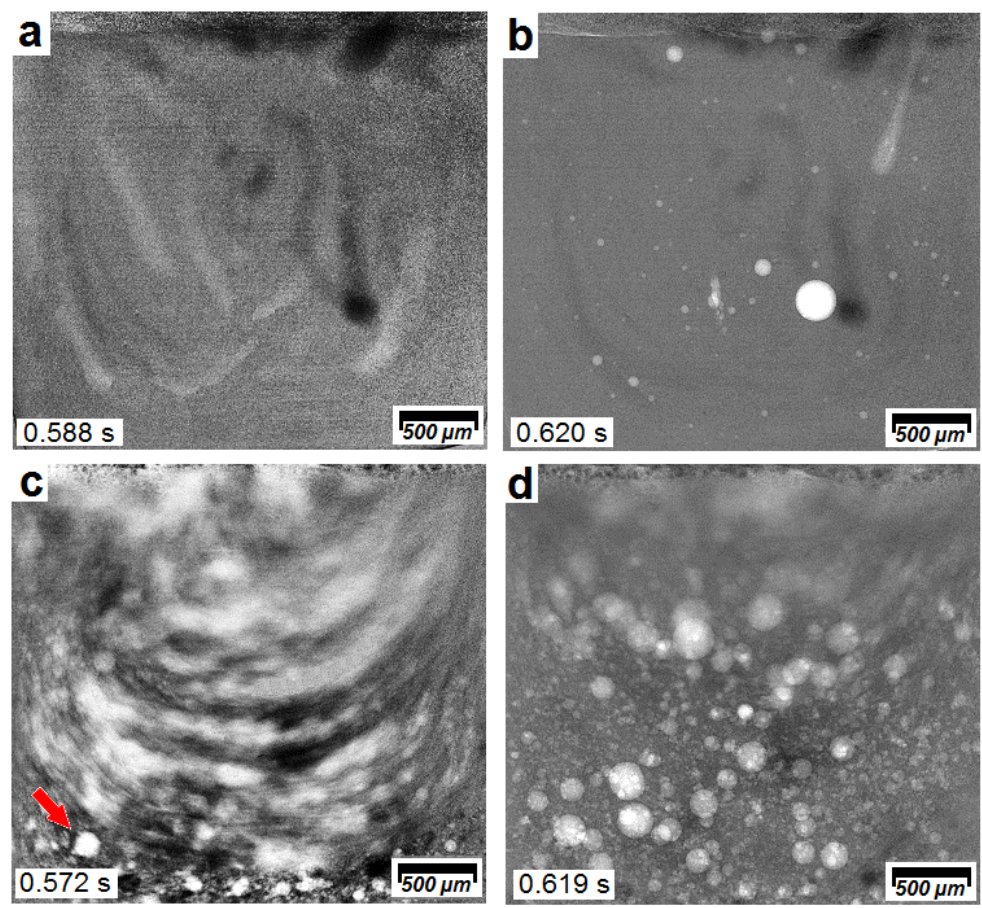

Figure 2. Radiographs of Al-10\%Cu sample without nano particles during: a) sonication, b) just after sonication is stopped; and $\mathrm{Al}-10 \% \mathrm{Cu}$ with nano particles added during c) sonication, and d) just after sonication is stopped. The red arrow shows a slow moving bubble.

Figure 2 shows representative radiographs obtained during the ultra-sonication of molten samples. Flow during the sonication periods was observed with and without NPs. Once the pressure difference is induced by the ultrasonic waves, cavitation process in liquids is usually initiated via heterogeneous nucleation of cavitation bubbles from pre-existing fine gas bubbles [4]. Alloy melts typically contain some non-wettable sub-micron sized solid particles/inclusions with gaseous phase associated with their surface defects, which provide a strong potential for cavitation nuclei [4]. During the sonication period, it was not possible to detect individual cavitation bubbles in most of the images, instead blurring effects cause the rapidly moving bubbles to appear as continuous streaks (Fig. 2.c and Supplementary video\#1). An exception was found for samples with NPs, some bubbles were visible without streaking in the lower bottom portions of the sample(s), example is shown by a red arrow Figure 2.c. At intermittent-no sonication periods, bubbles were clearly observed in the molten melts (Fig. 2.d and 
Supplementary video\#2), while bubbles move at speeds of over $100 \mathrm{~mm} / \mathrm{s}$ during sonication. When there is no-sonication, bubbles continue to move but at low speeds up to $\sim 3.0 \mathrm{~mm} / \mathrm{s}$, presumably as a result of residue flow momentum from sonication and buoyancy effects. It is to be noted that some of these residual bubbles are likely to be attached to the crucible walls, slows down their movement, preventing their floating to the surface. However, once the next sonication cycle starts, nearly all of the existing bubbles were annihilated instantly and a new set of bubbles appear at the end of the new sonication cycle. Apart from this dynamic behaviour; significantly higher number of bubbles were observed in the melt(s) containing NPs (Figs. 2.b vs. 2.d).

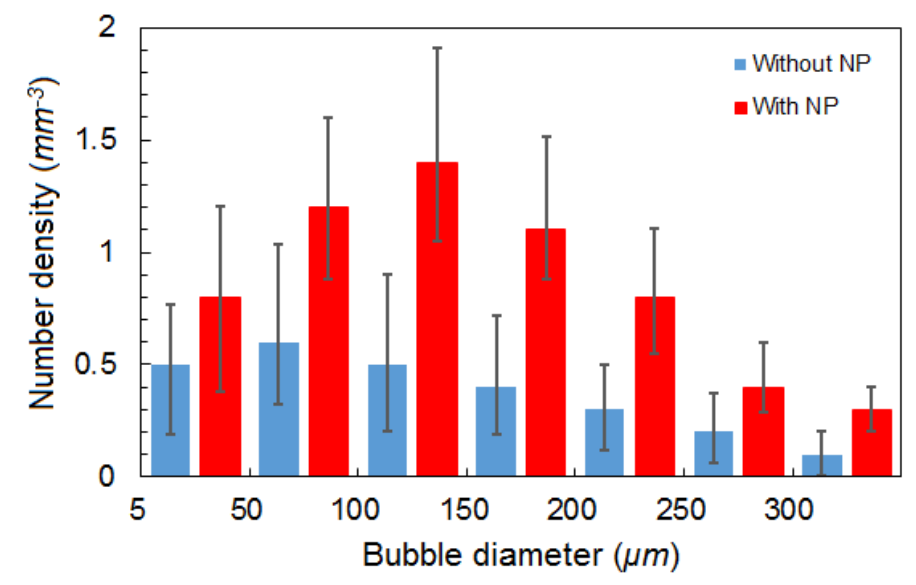

Figure 3. Bubble size distribution immediately after the sonication stops in a cycle for melts with and without NPs. Statistics are based on measurements from 10 different sonication cycles/experiments for each condition.

It appears that both bubble number density and the bubble average size increased in the melts with NPs as compared to the melt without NPs. The added $\mathrm{Al}_{2} \mathrm{O}_{3}$ particles (NPs) are generally not well wetted by liquid aluminium, and provide numerous cavities for entrained interfaces where atomic hydrogen from the melt can re-combine into molecular hydrogen. It is well known that particles with absorbed hydrogen act as cavitation nuclei, significantly reducing the cavitation threshold in liquid metals $[4,9]$. Thus, it is reasonable to hypothesise that $\mathrm{Al}_{2} \mathrm{O}_{3} \mathrm{NPs}$ are the main cause for the differences, through introducing numerous heterogeneous nuclei during cavitation. After sonication more bubbles are present with NPs, likely due to a greater initial number density. For quantification of this effect, we have extracted the bubble radius via adaptive threshholding (to separate the bubbles from the liquid 
background) and then by applying 2D Laplacian operator to detect the edges. Figure 3 presents quantified bubble diameter statistics as obtained from the radiographs. As shown in Figure 3, the bubble number density increases by two fold when NPs are present in the melt. In addition to the bubble density, there is a significant increase in the average bubble diameters, from 50-100 $\mu \mathrm{m}$ in the reference melt to $100-150 \mu \mathrm{m}$ in the alloy with NPs. This suggests that the growth and stability of cavitation gas bubbles was significantly enhanced in the presence of NPs. Remarkably, a possible clue on the reasons for the faster mass (hydrogen) transport, as a result of higher flow rates can also be found from image analysis, particularly in the upper half of the melts with NPs. As shown in Figure 2.a and c, a continuous streak indicates the projection of a bubble's movement during a single image exposure. Therefore the length of the each streak provide just a 2D projection of the bubble that it travels within $10 \mathrm{~ms}$. However, as we cannot extract any information about the distance travelled along the thickness direction, the streak lengths can only provide a rough approximation (in plane normal to the beam direction) as presented in Figure 4, rather than the exact flow velocity.

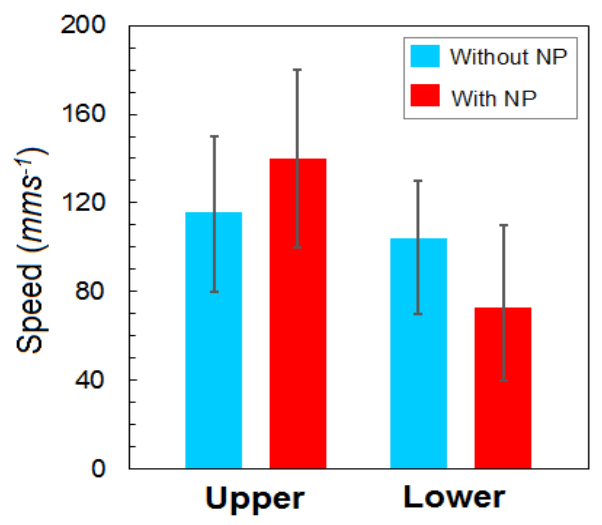

Figure 4. Flow speed estimation from bubble streaklines with and without nano particles. 'Upper' and 'Lower' denotes the measurements from upper and lower portions of the sample(s). Error bars indicate the range of the measured values, 10 readings for each case.

Figure 4 suggests that the average flow velocity is approximately $20 \%$ higher in the melts with NPs under the same sonication conditions, when the upper half of the crucible is considered. It is possible to view this higher velocity as an indication of increased turbulent nature of the MMNC melts, caused by greater cavitation bubble activity. The more active movement of cavitation bubbles may result in their accelerated growth due to the constantly refreshed supply of the hydrogen-saturated melt to the surface of the bubbles. In contrast to the flow rates closer to the sonotrode tip, marginally lower flow velocities 
were found for the bubble movement at a distance away from the sonotrode tip (the lower half of the observable melt). However, this difference is found to be higher for the cases with NPs. As shown in Figure 2c, the appearance of some distinguishable bubbles, from its circular shape (eg. one shown by the red arrow) provides further evidence for lower flow velocities. Note that the acoustic power attenuates further as cavitation bubbles form (shielding effect) [21]. This may further contribute to the appearance of relatively quiet bubbles at a distance from the ultrasonic source in the presence of cavitation enhanced by NPs.

Even though cavitation and acoustic streaming (agitation) is employed to improve solidification processing $[4,5]$, excessive agitation of melt has also been found to result in undesirable defects in castings, such as porosity [22], oxide bifilm formation [23] or detrimental morphological changes to the microstructure [24], resulting in weakened mechanical properties. Turbulence and agitation may also result in re-agglomeration of particles [25]. Further, it is to be noted that the flow velocities can effect segregation, depending on the local flow patterns. According to our observations and analysis presented here, ultrasonic driven melt processing of MMNCs can cause localisation of cavitation and flow dynamic effects within the crucible. Hence, ultrasonic processing of MMNCs is challenging when compared to conventional alloy melts.

\section{Conclusions}

Fast synchrotron radiographic images during in situ ultrasonication of $\mathrm{Al}-10 \mathrm{wt} \% \mathrm{Cu}$ alloys with/without added $\mathrm{Al}_{2} \mathrm{O}_{3}$ NPs were recorded to evaluate the cavitation potential during ultrasonic processing of MMNCs. Cavitation bubbles were quantified in terms of their influence on bubble velocity, number density and size distribution. It was found that the addition of NPs significantly intensify cavitation bubble formation and increase the melt agitation in the regions close to the sonotrode tip. However higher cavitation bubble volume with NPs tend to attenuate ultrasonic power propagation decreasing cavitation activity away from the sonotrode tip, introducing grater variation of ultrasonic effects across the MMNC melts when compared with conventional melts.

\section{Acknowledgements}

The authors wish to acknowledge financial support from the ExoMet Project, which is co-funded by the European Commission in the 7th Framework Programme (contract FP7-NMP3-LA-2012-280421), by the 
European Space Agency and by the individual partner organisations. UK EPSRC grants (EP/I02249X/1, EP/K00588X/1, EP/K005804) and the Research Complex at Harwell are also acknowledged. The authors gratefully acknowledge the use of the Diamond Manchester Branchline at Diamond Light Source (MT5001-2) and are grateful to Dr. Kaz Wanelik for implementing software scripts for imaging.

\section{References}

[1] H.H. Kim, J.SS. Babu, C.G. Kang, Materials Science and Engineering A 573 (2013) 92-99

[2] O. Liuzhang, L. Chengping, S. Xiandong, Z. Meiqin, Z. Min, Materials Letters 57 (2003) 17121715

[3] J.Q. Xu, L.Y. Chen, H. Choi, X.C. Li, Journal of Physics: Condensed Matter 24 (2012) 255304

[4] D. E. Eskin, G.I. Eskin, "Ultrasonic Melt Treatment of Light Alloys Melts", 2nd Edn.(2014), Hoboken, CRC Press

[5] G. Cao, H. Konishi, X. Li, Jounral of Manufacturing Science and Engineering 130 (2008) 031105

[6] X. Liu, Y. Osawa, S. Takamori, T.ji Mukai, Materials Letters 62 (2008) 2872-2875

[7] R. Kumar, D. Milanova, Applied Physics Letters 94 (2009) 073107

[8] S. Vafaei, A. Purkayastha,A. Jain,G. Ramanath, T. Borca-Tasciuc, Nanotechnology 20(2009) 185702

[9] W. Lauterborn, W. Hentschel, Ultrasonics (1985) 260-268

[10] P.D. Lee, J. Hunt, Scripta Materialia 36(1997) 399-404

[11] R. H. Mathiesen, L. Arnberg, H. Nguyen-Thi, B. Billa, JOM 64 (2012) 74-82

[12] B. Cai, S. Karagadde, L. Yuan, T.J. Marrow, T. Connolley, P.D. Lee, Acta Materialia 76 (2014) 371-380

[13]W.U. Mirihanage, M. Di Michiel, A. Reiten, L. Arnberg , H.B. Dong, R.H. Mathiesen, Acta Materialia 68 (2014) 159-68

[14] A.G. Murphy, W.U. Mirihanage , D.J. Browne, R.H. Mathiesen, Acta Materialia 95 (2015) 83-89

[15]A.G. Murphy, D.J. Browne, W.U. Mirihanage, R.H. Mathiesen, Acta Materialia 61(2013) 45594571

[16] H.J. Huang, D. Shu, Y.A. Fu, J. Wang, B.D. Sun, Ultrasonic Sonochemistry 21 (2014) 1275-1278 $\mathrm{S}$

[17] T. Nagira, N. Nakatsuka, H. Yasuda, K. Uesugi, Materials Letters 150 (2015) 135-138

[18] D. Tan, T.L. Lee, J.C. Khong, T. Connolly, K. Fezza, J. Mi,Metallugical Mater. Trans. A 46 (2015) 2015-2851

[19] H.Huang, D. Shau, J. Zeng,F. Bian, Y. Fu, J Wang, B. Sun, Scripta Materialia 106 (2015) 21-25 A [20] D. Fanis, Z. D. Pesic, U. Wagner, C. Rau, Journal of Physics: Conf. Series. 425 (2013) 192014

[21] L.D. Rozenberg, "Powerful Ultrasonic Fields" - Part VI Cavitation Region (1968), Nauka, Moscow, 221-266

[22] R.C. Atwood, S. Sridhar, P.D. Lee, Scripta Materialia 41(1999) 1255-1259

[23]D. Dispinar, S. Akhtar, A. Nordmark, M. Di Sabatino, L. Arnberg, Materials Science and Engineering A 527 (2010) 3719-3725 
[24] Y. Zhang, J. Jie, Y. Gao, Y. Lu, T. Li, Intermetallics 42 (2013) $120 \mathrm{e} 125$

[25] M. Afkhami, A. Hassanpour, M. Fairweather, D.O. Njobuenwu, Computer Aided Chemical Engineering 33 (2014) 967-972 